\title{
Successful control of outbreak caused by clonally related extensively- resistant Acinetobacter baumannii in an intensive care unit
}

\author{
Bushra Sultan ${ }^{1}$, Aamer Ikram ${ }^{1}$, Bruno Lopes ${ }^{2}$, Ziaullah Jarral ${ }^{1}$, Muhammad Yasir Rafiq ${ }^{1}$, Gohar Zaman ${ }^{1}$, \\ Parvez Asghar ${ }^{3}$, Muhammad Ayyub ${ }^{1}$ \\ ${ }^{1}$ Department of Microbiology, Armed Forces Institute of Pathology, National University of Medical Sciences, \\ Rawalpindi, Pakistan \\ ${ }^{2}$ Department of Microbiology, University of Aberdeen, United Kingdom \\ ${ }^{3}$ Medical Intensive Care Unit, Military Hospital, Rawalpindi, Pakistan
}

\begin{abstract}
Objective: Worldwide drug resistance in Acinetobacter baumannii is on the rise. We report outbreak of extensivelyresistant (XDR) $A$. baumannii in a medical intensive care unit (MICU) at a tertiary care referral hospital in Pakistan. During subsequent survey, 10 extensively-resistant $A$. baumannii were isolated from 8 environmental samples and hands of 2 healthcare workers (HCWs).
\end{abstract}

Methods: In January 2013, XDR A. baumannii (Ab1, Ab2, and Ab3) were isolated from tracheal aspirate, blood and sputum of three patients with ventilator associated pneumonia. The isolates were resistant to piperacillin, ampicillinsulbactam, piperacillin-tazobactam, ticarcillin-clavulanic acid, ceftazidime, cefipime, ceftriaxone, imipenem, meropenem, gentamicin, amikacin, doxycycline, minocycline, ciprofloxacin and trimethoprim-sulphamethoxazole.. Pulsed Field Gel Electrophoresis (PFGE) revealed that isolates from HCWs were similar to the genotype initially isolated from patients' samples.

Results: Control of the outbreak was attained with requisite infection control practices and fumigation of the Medical ICU unit. From February 2013 to April 2013 there were no new cases of extensively-resistant isolates in medical ICU. However, two more cases with similar antibiogram MICs and genotype of extensively-resistant isolates emerged in June 2013 from the same MICU.

Conclusion: Stringent infection control measures were implemented this time with continuous monitoring and regular surveillance. Follow up for the next two years has been successful as no clustering of XDR $A$. baumannii were detected from medical and surgical ICUs. J Microbiol Infect Dis 2017; 7(1): 1-6

Keywords: Hospital Infection Control, health care workers, surveillance

\section{INTRODUCTION}

Acinetobacter spp. has become a notorious threat for hospitalized patients especially those on mechanical ventilation. They may be part of the bacterial flora of moist regions of skin like axilla and groin; the carrier rate increases in hospitalized patients more so in outbreaks [1]. Higher colonization rates of the potent areas of the body like skin, throat, respiratory tract and digestive tract have been documented [2]. Consequently contamination of hands of HCWs occurs even after minor contact with colonized patients [3]. The organism has the potential to persevere in hospital milieu for many days [3].
Additional dilemma related to $A$. baumannii is the emergence of resistance to multiple antibiotics [4]. Many Acinetobacter spp. are resistant to frequently used antibiotics like aminopencillins, ureidopencillins, cephalosporins and aminoglycosides. Carbapenems, betalactam/beta-lactamase inhibitor combinations and tetracyclines like minocycline and tigecycline and colistin are the commonly used against severe $A$. baumannii infections, however resistance against these is also on steady rise [5].

Outbreaks caused by multidrug resistant (MDR), XDR and pan-resistant $A$. baumannii have been 
reported $[4,5]$. Risk factors associated with the resistance acquisition mainly include injudicious and broad spectrum antibiotic exposure, prolonged stay in the ICUs, mechanical ventilation and drifting from local antibiogram based antibiotic policies [6]. Investigations of several outbreaks of resistant infections have been ascribed to direct contact [7]. We describe outbreak of $A$. baumannii that occurred in a newly commissioned ICU of a major operation theatre complex at a tertiary care hospital.

\section{METHODS}

The study was conducted at Armed Forces Institute of Pathology (AFIP), Rawalpindi, Pakistan, from January 2013 through January 2015. It sup-ports 1000-bedded tertiary care teaching hospital with two ICUs for surgical and medical patients and a high dependency unit (HDU). MICU is a 15-bedded newly constructed unit inducted in 2012. The ICUs and the HDU are on the same floor but independent units. The study was approved by the Review Board and Ethical Committee of the institute.

\section{Case definition}

Patient with at least one clinical sample yielding A. baumannii resistant to beta-lactam group or its combinations, aminoglycosides, tetracyclines sulphamethoxazole-trimethoprim and quinolones was defined as a case of infection or colonization only if sample yielding the isolate was taken 48 hours post ICU admission. Hospital acquired infections were recognized as per Centers for Disease Con-trol and Prevention (CDC) criteria [8]. Isolates that were resistant to piperacillin, ceftazidime, ceftriaxone, cefipime, imipenem, meropenem, tazobactam-piperacillin, ampicillin-sulbactam, sulbactam-cefoperazone, ciprofloxacin, cotrimoxazole, doxycycline and minocycline were labeled as XDR.

Index case (Isolate-Ab1) was the first patient from whom XDR $A$. baumannii was isolated; a 52 -years-old male initially hospitalized in the general ward (January 5, 2013) for ten days and then shifted to the MICU with melena, septic shock and lobar pneumonia followed by tracheostomy. He was on intravenous meropenem and linezolid; as the condition deteriorated intravenous colimycin but this did not help.
Second case (Isolate-Ab2), was a 70-years-old male with the longest stay in the ICU from September 2012 till February 2013. He presented with Non-Hodgkin's Iymphoma; developed lower respiratory tract infection and sepsis after tracheostomy. Third case (IsolateAb3), was from 57-years-old male who suffered stroke (January 21, 2013); after decompression craniotomy, he was also put on tracheostomy support.

All the three patients had fever and succumbed to death on January 24, 27 and 29, 2013 respectively. The patients were looked after by the same group of doctors and nursing staff. No case was reported or detected during the next couple of months i.e., March and April 2013. However, two cases were identified in the month of June 2013; a 65-years-old female with full form of cerebrovascular accident and aspiration pneumonia, and a 36-years-old male with head injury. Both were on ventilator support; only the second case survived. Follow up for two years has been successful and no new case of XDR A. baumannii has been detected. The outbreak curve is shown in Figure 1.

\section{Sample and data collection}

Phenotypic and genotypic determination of the isolates collected in the outbreak was carried out by a team from the Microbiology Department, AFIP. Medical records of the patients were reviewed for clinical information including age, gender, and hospital location before shifting to ICU, length of stay, laboratory reports, antimicrobials used, and ventilator support. Our infection control team conducted sampling from the entire unit. As mentioned in Table 1, on February 1, 2013, sampling was conducted for tracing the XDR species; in total 75 swabs from various inanimate objects, along with endotracheal tubes of concerned patients. Swabs from the environmental sources and the hands of healthcare workers were included. The samples were inoculated onto $5 \%$ sheep blood agar plates and incubated overnight at $37^{\circ} \mathrm{C}$. Identification of organisms yielded was through standard tests and applying API 20NE (bio Merieux, France). A. baumannii identification and isolation was done as per standard procedures and by $\mathrm{rpoB}$ sequencing as mentioned earlier by Lopes et al [9]. Antibiotic disk susceptibility testing and MICs using the 
agar dilution method were performed as per the Clinical Laboratory Standards Institute (CLSI) guidelines [10]. The antibiotic discs (Oxoid, UK) for susceptibility testing were: amikacin $(30 \mu \mathrm{g})$, ampicillin-sulbactam $(10 / 10 \mu \mathrm{g})$, ceftriaxone $(30$ $\mu \mathrm{g})$, ceftazidime $(30 \mu \mathrm{g})$, cefipime $(30 \mu \mathrm{g})$, ciprofloxacin $(5 \mu \mathrm{g})$, doxycycline $(30 \mu \mathrm{g})$, gentamicin $(10 \mu \mathrm{g})$, imipenem $(10 \mu \mathrm{g})$, meropenem $(10 \mu \mathrm{g})$, minocycline $(30 \mu \mathrm{g})$, piperacillin (100 $\mu g)$ piperacillin-tazobactam (100/10 $\mu g)$, ticarcillin-clavulanic acid $(75 / 10 \mu \mathrm{g})$, and trimethoprim-sulphamethoxazole $\quad(1.25 / 23.75$ $\mu \mathrm{g})$. As CLSI did not offer susceptibility criteria for tigecycline, zone of inhibition was reported.

\section{PFGE Methods}

Apa I macro-restriction typing PFGE was employed for identifying the epidemiological correlation of the isolates according to the procedure described by Seifert et al [8]. The gels were stained with ethidium bromide solution. Diversity Database software image capturing system (Bio-Rad Laboratories Ltd, UK) was used for analysis. Criteria described earlier by Tenover et al. were applied for inferring the clonal correlation [11].

\section{Infection Control Measures}

During the first episode, outbreak control strategies were revisited and implemented. Staff awareness and training was ensured. Hand washing was prioritized. Screening of the staff members was conducted. Fumigation was done with hydrogen peroxide. Surface cleaning was monitored, and appropriate disinfection practices were implemented for equipment like ventilators, suction machines, etc.

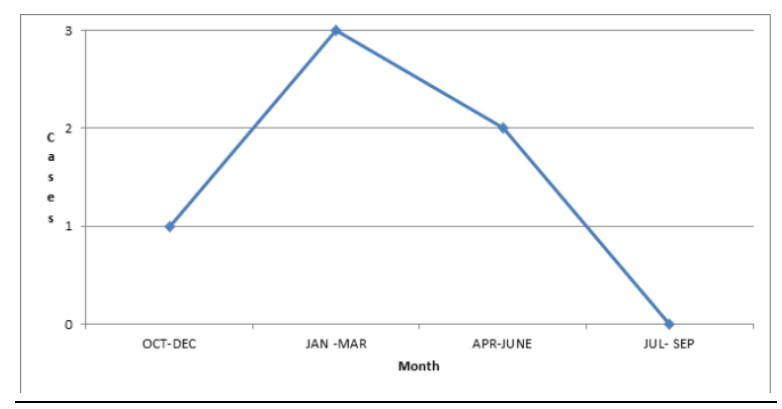

Figure 1. The outbreak curve.

Despite these measures, new resistant cases surfaced after a short span of two months. This time a dedicated team was detailed including a microbiologist and infection control specialist. Regular surveillance was done. Strict infection control practices were stringently implemented. Movement in and around the ICUs was restricted. Staff were not only regularly guided but monitored as well. Mechanical ventilation equipment was supervised. Limitation of the visitors was strictly implemented. The XDR cases dropped but a couple of cases persisted in the MICU despite the efforts. At that time, the infection control committee decided to evacuate the entire ward and fumigated with formaldehyde to properly eradicate the environmental source of the infection. Patients were put into two cohorts (con-tact patients and newly admitted patients). Strict precautions including contact isolation were implemented to prevent the hand borne transmission through HCWs. No one was allowed to attend the patients in ICUs without proper PPE. Environmental contamination was prevented by cleaning all surfaces of the ward by using sodium hypochlorite. Disinfection of all bed sites with alcohol, and floors and sinks with sodium hypochlorite was ensured. Endotracheal suction was monitored to prevent transmission and environmental contamination. Significance of proper hand hygiene was frequently highlighted to various tiers. Open suctioning was discouraged and closed suction systems were employed. Appropriate antimicrobial usage, based on the local antibiogram, was ensured in the ICUs.

\section{RESULTS}

During the outbreak period, out of 75 screening swab samples, 10 specimens yielded XDR $A$. baumannii (Table 1). Among the $14 \mathrm{HCWs,} 2$ staff members tested positive for XDR $A$. baumannii. Following the outbreak, the screening swabs yielded 16 A. baumannii isolates, of which $10(62 \%)$ had MICs that matched the MICs of index case Pipracilin (128 $\mu \mathrm{g} / \mathrm{ml})$, ampicillin-sulbactam $(32 / 16 \mu \mathrm{gg} / \mathrm{ml})$, piperacillin-tazobactam $(128 / 4 \mu \mathrm{g} / \mathrm{ml})$, ticarcillinsulbactam $(128 / 2 \mu \mathrm{g} / \mathrm{ml})$, ceftazidime $(64 \mu \mathrm{g} / \mathrm{ml})$, cefepime $(32 \mu \mathrm{g} / \mathrm{ml})$, ceftriaxone $(128 \mu \mathrm{g} / \mathrm{ml})$ imipenem $(64 \mu \mathrm{g} / \mathrm{ml})$, meropenem $(32 \mu \mathrm{g} / \mathrm{ml})$, gentamicin $(32 \mu \mathrm{g} / \mathrm{ml})$, amikacin $(64 \mu \mathrm{g} / \mathrm{ml})$, doxycycline $(32 \mu \mathrm{g} / \mathrm{ml})$, minocycline $(16 \mu \mathrm{g} / \mathrm{ml})$, ciprofloxacin (8 $\mu \mathrm{g} / \mathrm{ml})$, trimethoprimsulphamethoxazole $(4 / 76 \mu \mathrm{g} / \mathrm{ml})$, polymixin B (1 
$\mu \mathrm{g} / \mathrm{ml})$. Figure 2 shows the genotypic relatedness of the initial cases and the phenotypic similar isolates from screening sampling. Out of the hand samples from 14 HCWs, 2 of the (one nurse and one nursing aid) yielded XDR $A$. baumannii. Average length of stay of the patients in the ICU was 55 days.

Table 1: Samples of XDR isolates detected.

\begin{tabular}{lcc}
\hline Sampling site & $\begin{array}{c}\text { No. of } \\
\text { samples }\end{array}$ & $\begin{array}{c}\text { XDR A. } \\
\text { baumannii } \\
\text { cases (n) }\end{array}$ \\
\hline HCWs (n=14) & 2 & Nil \\
\hline Doctors & 7 & 1 \\
Nurses & 5 & 1 \\
Nursing aides & & \\
\hline Environmental & 17 & 3 \\
samples (n=61) & 16 & 2 \\
\hline Bedding & 4 & Nil \\
Side tables & 6 & Nil \\
IV sets & 5 & Nil \\
Mechanical \\
ventilators
\end{tabular}

According to the Kirby-Bauer disk diffusion method, all $A$. baumannii isolates were resistant to ampicillin-sulbactam, piperacillin-tazobactam, ticarcillin-sulbactam, imipenem, meropenem, gentamicin, amikacin, doxycycline, minocycline, ciprofloxacin, trimethoprim-sulphamethoxazole. The isolates were sensitive only to polymyxin B by MICs conducted as per CLSI guidelines [10]. PFGE patterns showed that a total of 13 isolates recovered from the patients, hands of HCWs, and ward environments were related to each other according to Tenover's criteria [11]. The number of newly diagnosed declined with implementation of proper actions. These radical measures helped to eliminate the environmental source of $A$. baumannii infection in the affected ward. With persistent simple and stringent infection control practices like hand washing by the staff including nurses, doctors, paramedics and care takers before and after touching the patients, surface cleaning with $1 \%$ hypochlorite, provision of hand sanitizers with every bed, trained staff and reducing visitors, elimination of XDR A. baumannii was ensured.

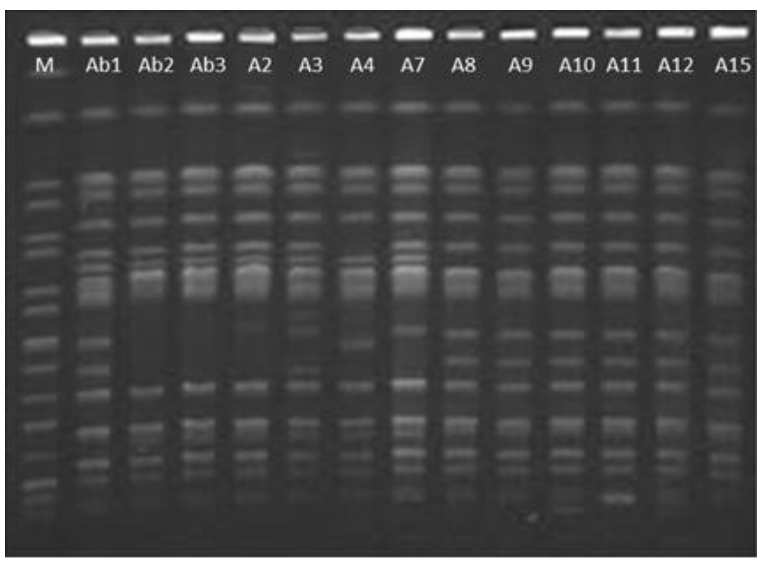

Figure 2. PGFE showing genetically related isolates (Ab1-Ab3) from three initial cases, ten environmental samples including isolates from the hands of the healthcare workers (A2, A3, A4, A7, A8, A9, A10, $A 11, A 12, A 15)(M=$ Ladder $)$.

\section{DISCUSSION}

Over the recent years, Acinetobacter species have gained tremendous importance because of potential spread in hospital environments and more so among patients on mechanical ventilation. It has been increasingly implicated in colonization and infection of patient admitted in hospitals [12]. Due to it fluid genome, this bacterium has the ability to attain rapid resistance to most of the commonly employed antimicrobials. The problem intensifies as it can colonize or spread among already moribund patients. The effective control of hospital acquired infections caused by XDR bacteria has been a critical concern not only for developing world but for the developed countries as well. Infection with this organism can present in a variety of ways particularly pneumonia associated with ventilators and resuscitators.

The outbreak reported in present study may be due inadequate implementation of infection control procedures with consequential transmission as has been documented earlier [13]. Environmental contamination has been responsible for spread of pan-resistant $A$. baumannii strains in ICUs; hands being the most important source for transmittance [14]. Another 
associated problem with Acinetobacter species is persistence on inanimate objects thus facilitating colonization and spread [15]. In the present study, molecular testing confirmed same genotype of the isolates from the clinical, environmental and HCWs' hands. This clearly indicated that the patient to patient transmission occurred as a result of contact.

Clinically, ventilator associated pneumonia was associated with XDR $A$. baumannii infection. Closed-suctioning system minimizes environmental contamination and was thus preferred as suggested previously [16]. Some studies have reported complete sealing of units for control the outbreaks $[15,16]$. In our case, during the initial outbreak, ICUs were not closed due to huge dependency. However, the second episode was alarming and demonstrated that closure of the contaminated units was pivotal. Fumigation of closed unit was done and strict contact precautions were implemented.

The finding of strains showing high degree of relatedness was in agreement with earlier reports; however polyclonal outbreaks coexisting with the epidemic strains have also been reported [17]. Further analyses are mandatory to search for allied causes.

Increasing antibiotic resistance detected in $A$. baumannii spp. poses serious problems in treatment for nosocomial infection caused by this pathogen. Moreover, ever-increasing use of antimicrobials favors the emergence and spread of MDR A. baumannii in the hospital settings [18]. Con-trolled and judicious use of antibiotics is thus mandatory to prevent epidemic $A$. baumannii infections.

\section{Conclusion}

A. baumannii can spread widely though colonized persons and contaminated inanimate objects and environments. Proper hospital infection control strategies are to be strictly implemented and stringently observed for avoiding outbreak of $A$. baumannii. The study emphasizes the fact that infection control measures require a multifaceted approach with multi-factorial implementation of specific control measures including hand washing, surface cleaning, and adherence to infection control precautions together by the HCWs, patients and care givers. Environmental decontamination, surface cleaning and specific use of a disposable closed-suction system for intubated patients has proved rewarding. Keeping dedicated staff responsible for not only observing the infection control practices stringently but also training staff is beneficial. Maintenance followed by surveillance is the best method to keep track of infection control practices and identification of the loop holes causing hospital acquired infections. Such measures will greatly improve hospital infection control and eradication of $A$. baumannii in the hospital environments.

Declaration of conflicting interests: The authors declared no conflicts of interest with respect to the authorship and/or authorship of this article.

Funding: The authors received no financial support for the research and/or authorship of this article.

\section{REFERENCES}

1. Peleg AY, Seifert H, Paterson DL. Acinetobacter baumannii: emergence of a successful pathogen. Clin Microbiol Rev 2008; 21: 538-582.

2. Durante-Mangoni E, Zarrilli R. Global spread of multi-drug-resistant Acinetobacter baumannii: molecular epidemiology and management of antimicrobial resistance. Future Micro-biol 2011; 6(4): 407-422.

3. Kramer A, Schwebke I, Kampf G. How long do nosocomial pathogens persist on inanimate surfaces? A systematic review. BMC Infect Dis 2006; 6:130.

4. Aygun G, Demirkiran O, Utku $T$, et al. Environmental contamination during a carbapenem-resistant Acinetobacter bau-mannii outbreak in an intensive care unit. J Hosp Infect 2002; 52:259-262.

5. Levin AS, Gobara S, Mendes CM, Cursino MR, Sinto S. Environmental contamination by multidrug-resistant Aci-netobacter baumannii in an intensive care unit. Infect Con-trol Hosp Epidemiol $2001 ; 22: 717-720$

6. Lee NY, Lee HC, Ko NY, et al. Clinical and economic impact of multidrug resistance in nosocomial Acinetobacter baumannii bacteremia. Infect Control Hosp Epidemiol 2007; 28: 713-719.

7. Kwon KT, Oh WS, Song JH, et al. Impact of imipenem resistance on mortality in patients with Acinetobacter bacteraemia. J Antimicrob Chemother 2007; 59:525-530. 
8. Seifert H, Dolzani L, Bressan R. Standardization and interlaboratory reproducibility assessment of Pulsed-Field Gel Electrophoresis generated fingerprints of Acinetobacter baumannii. J Clin Microbiol 2005; 43(9): 4328-4335.

9. Lopes BS, Al-Hassan L, Amyes SGB. ISAba825 controls the expression of the chromosomal b/aOXA-51-like and the plasmid borne blaOXA-58 gene in clinical isolates of Aci-netobacter baumannii isolated from the USA. Clin Microbiol Infect 2012; 18(11):E446-451.

10. Clinical and Laboratory Standards Institute. Performance standards for antimicrobial susceptibility testing approved standard 24th ed. Wayne, PA: CLSI: 2014 [DocumentM100-S24].

11. Tenover FC, Arbeit RD, Goering RV, Mickelsen PA, Murray BE, Persing DH, Swaminathan B. Interpreting chromosomal DNA restriction patterns produced by pulsed-field gel elec-trophoresis: criteria for bacterial strain typing. J Clin Micro-biol 1995; 33:2233-2239.

12. Markogiannakis A, Fildisis G, Tsiplakou S, et al. Cross-transmission of multidrug-resistant Acinetobacter baumannii clonal strains causing episodes of sepsis in a trauma intensive care unit. Infect Control Hosp Epidemiol 2008; 29:410-417.

13. Wybo I, Blommaert L, De Beer T, et al. Outbreak of multidrug-resistant Acinetobacter baumannii in a Belgian university hospital after transfer of patients from Greece. J Hosp Infect 2007; 67:374380.

14. El Shafie SS, Alishaq M, Leni Garcia M. Investigation of an outbreak of multidrug-resistant Acinetobacter baumannii in trauma intensive care unit. J Hosp Infect 2004; 56:101-105.

15. Playford EG, Craig JC, Iredell JR. Carbapenemresistant Acinetobacter baumannii in intensive care unit patients: risk factors for acquisition, infection and their consequences. J Hosp Infect 2007; 65:204-211.

16. Zanetti G, Blanc DS, Federli I, et al. Importation of Acinetobacter baumannii into a burn unit: a recurrent outbreak of infection associated with widespread environmental contamination. Infect Control Hosp Epidemiol 2007; 28:723-725.

17. D'Agata EM, Thayer V, Schaffner W. An outbreak of Aci-netobacter baumannii: the importance of cross-transmission. Infect Control Hosp Epidemiol 2000; 21:588-591.

18. Zarrilli R, Pournaras S, Giannouli M, Tsakris A. Global evo-lution of multidrug-resistant Acinetobacter baumannii clonal lineages. Int $\mathrm{J}$ Antimicrob Agents 2013; 41(1): 11-19. 